\title{
A Powerful Two-Stage Association Test Using Case-Control and Case-Parents Genotype Data
}

\author{
K.F. Cheng ${ }^{a, b}$ W.J. Lin ${ }^{b} \quad$ J.H. Chen ${ }^{a} \quad$ J.T. Horng ${ }^{c}$ \\ ${ }^{a}$ Biostatistics Center and Department of Public Health, China Medical University, ${ }^{b}$ Graduate Institute of Statistics, \\ National Central University, and ${ }^{\mathrm{C} D e p a r t m e n t}$ of Computer Science and Information Engineering, National Central \\ University and Department of Bioinformatics, Asia University, Taichung, Taiwan, ROC
}

\section{Key Words}

Family-based study · Population-based study • Population stratification

\begin{abstract}
Objective: The performance of association tests based on case-control or case-parents substudy alone can be improved by jointly using genetic data from two substudies. However, genetic data from different sources may not be combinable due to population stratification. We propose a two-stage association test based on using combinability tests in stage 1 and association tests in stage 2. Methods: The combinability tests are designed for testing that genotype data from different sources have same genotype frequencies and relative risks. The association tests are well known tests in the literature. We propose a method to adjust the significance levels at two stages so that the overall type I error rate of the two-stage test can be controlled at the desired level. Results: The simulation results confirm that the two-stage test has empirical type l error rates approximately equal to the predetermined levels while making substantially fewer false negatives than the usual test based only on case-parents substudy. Conclusion: It is advantageous to combine case-control and case-parents data into a single analysis. The two-stage test has significant power improve-
\end{abstract}

ment when the family-based test has weak or moderate power performance and is robust to the effect of population stratification.

Copyright $\odot 2009$ S. Karger AG, Basel

\section{Introduction}

Association tests for genetic markers and disease usually are constructed for comparing frequencies of genotypes from a sample of diseased individuals with those from a certain sample of controls. In a population-based case-control study, the sample of controls is a collection of unrelated nondiseased individuals. This study is often criticized because of the possible confounding effect of population stratification. This effect often leads to spurious associations and bias will not go away by increasing sample size.

To avoid the confounding effect of population stratification, some authors [1-6] proposed an intuitively appealing solution by measuring the genetic marker on both the disease cases and their parents. They suggested using transmitted parental alleles as a case sample and nontransmitted parental alleles as a control sample so that the cases and controls are matched in genetic ancestry and thus the effect of population stratification can be 
removed. Well known tests based on this idea include the transmissiom/disequilibrium test (TDT) [2] and the test based on the conditional on parental genotypes likelihood (CPG) [7]. The relative advantages and disadvantages of the family-based and population-based designs were discussed in Mitchell [8], Nagelkerke et al. [9], and Epstein et al. [10], etc.

Nagelkerke et al. [9] and Epstein et al. [10] considered a design where samples of genotype data of parents and their diseased offspring and genotype data from unrelated cases and controls are collected. This type of data may arise, for example, when a population-based casecontrol study was conducted first and a confirmatory family-based study was included later. When there is no effect of population stratification (or equivalently, the data are combinable), they showed that their tests, based on the combined data from family triads and unrelated subjects, are more powerful comparing with the usual tests based only on either the case-control substudy or the case-parents substudy alone. Ackerman et al. [11] also pointed out that the combined study design is most costeffective when cases are difficult to ascertain and, when the case-control and TDT results agree, offers greater confidence in the result. Epstein et al. also suggested that if the data are not combinable, then the usual CPG approach should be used for testing gene-disease association.

While the method of Epstein et al. [10] is powerful for studying gene-disease association, their approach is in effect a two-stage approach that involves correlated tests in the testing procedure. Unfortunately, one still has no guideline on choosing significance level at each stage of their test so that the overall significance level can achieve the predetermined level. In this paper, our main focus is to suggest a novel two-stage test (denoted as TS) based on using two combinability tests at the first stage and the association tests of Epstein et al. at the second stage. To control the overall type I error rate of the TS test, we also have suggested an effective strategy for adjusting the significance levels for the tests at two stages. This strategy ensures that the probability of making any false positive is approximately equal to the predetermined level. The TS test is robust to the genetic model and effect of population stratification. In the case of missing parental genotypes, one also can easily apply existing methods to modify the TS test to account for the missing data.

We have conducted a simulation study to compare the performance of the TS test with the CPG test. Simulation results show that under different (none to moderate) levels of population stratification, all empirical type I error
Table 1. Conditional probability $P\left(G=g \mid G_{p}=g_{p}, D=1\right)$ for the CPG approach

\begin{tabular}{llll}
\hline $\begin{array}{llll}\text { Genotype }(G) \\
G_{p}\end{array}$ & $\begin{array}{l}A A \\
(2,2)\end{array}$ & $\begin{array}{l}A a \\
1\end{array}$ & $\begin{array}{l}a a \\
(2,1)\end{array}$ \\
\hline$\frac{\varphi_{2}}{\varphi_{1}+\varphi_{2}}$ & $\frac{\varphi_{1}}{\varphi_{1}+\varphi_{2}}$ & 0 \\
$(2,0)$ & 0 & 1 & 0 \\
$(1,1)$ & $\frac{\varphi_{2}}{1+2 \varphi_{1}+\varphi_{2}}$ & $\frac{2 \varphi_{1}}{1+2 \varphi_{1}+\varphi_{2}}$ & $\frac{1}{1+2 \varphi_{1}+\varphi_{2}}$ \\
$(1,0)$ & 0 & $\frac{\varphi_{1}}{1+\varphi_{1}}$ & $\frac{1}{1+\varphi_{1}}$ \\
$(0,0)$ & 0 & 0 & 1
\end{tabular}

rates of the TS test are very close to the expected type I error rates. This shows that the TS test is robust to the effect of population stratification. General simulation results also show that the TS test is more powerful. In particular, when the CPG test has weak or moderate power performance, the improvement of the TS test is more pronounced. However, when the number of family triads becomes large, the improvement decreases.

\section{Material and Methods}

We assume to have a sample consisting of triads, unrelated cases and controls that are all genotyped at the marker of interest. The numbers of triads, unrelated cases and controls are respectively equal to $N_{f}, N_{1}$ and $N_{0}$. G denotes the subject's genotype, representing the number of copies of the high-risk allele carried, and $G_{p}=\left(G_{1}, G_{2}\right)$ denotes his/her parental genotypes with the convention that $G_{1} \geq G_{2}$. The parameter $\varphi_{g}$ is defined to be the relative risk of the subjects with genotype $G=g$ versus those with base-line genotype $G=0$. In the discussion of population stratification, we follow the CPG approach to assume that mating symmetry is satisfied within each subpopulation. Finally, we let $D$ denote the disease outcome of a subject $(1=$ diseased, and $0=$ undiseased).

In family-based study, a popular association test is defined to be the likelihood ratio test of no association, $H_{0}: \varphi_{1}=\varphi_{2}=1 \mathrm{using}$ CPG likelihood:

$$
L_{f}\left(\varphi_{1}, \varphi_{2}\right)=\prod_{i=1}^{N_{f}} P\left(G_{i}=g_{i} \mid G_{p i}=g_{p i}, D_{i}=1\right) .
$$

Here, $P\left(G=g \mid G_{p}=g_{p}, D=1\right)$ is given by Schaid and Summer [7] under the assumptions that offspring disease risk is independent of parental genotype given offspring genotype and Mendelian proportions holds; see also table 1 . The CPG approach is robust to 
the effect of population stratification if these two assumptions are also true within each subpopulation. Under the null hypothesis, the CPG test, $T_{C P G}$, has chi-square distribution with 2 degrees of freedom. We denote the $\mathrm{p}$ value of the CPG test as $P_{C P G}$.

\section{The First Stage Tests: Testing the Combinability of Genotype}

Data from Family Triads and Unrelated Subjects

In the following, we outline the combinability tests proposed in this paper. The details for deriving these tests are given in the Appendix. Note that according to the argument in Epstein et al. [10], the genotype data from triads and unrelated subjects can be combined for association analysis only when the genotype data from different sources were sampled from the populations having same genotype frequencies and relative risk parameters. Thus, the following combinability tests are in essence constructed for testing the absence of the effect of population stratification between different data sources.

First, the result in the Appendix shows that the genotype data from triads and unrelated controls are combinable if the null hypothesis $H_{0}^{c o}: \beta_{1}=\log \hat{\varphi}_{1}, \beta_{2}=\log \hat{\varphi}_{2}$, based on the likelihood ratio test and likelihood function

$$
L\left(\beta_{1}, \beta_{2}\right)=\prod_{j=1}^{N_{0}+N_{f}} \frac{1}{1+\exp \left(\mu+\beta_{g j}\right)} \prod_{k=1}^{N_{f}} \exp \left(\mu+\beta_{g k}\right),
$$

is not rejected. Here $\beta_{i}$ are log-odds-ratio parameters and $\mu$ is a normalizing constant defined in the Appendix. $g j(g k)$ are genotypes of the diseased children and unrelated controls (diseased children) and $\left(\hat{\varphi}_{1}, \hat{\varphi}_{2}\right)$ are the maximum likelihood estimates using CPG likelihood function $L_{f}\left(\varphi_{1}, \varphi_{2}\right)$. We denote the $\mathrm{p}$ value of this likelihood ratio test as $P_{\text {Com }}^{c o}$.

Next, the argument in the Appendix shows that the genotype data from triads and unrelated cases can be safely combined if the null hypothesis $H_{0}^{c a}: \beta_{1}^{*}=0, \beta_{2}^{*}=0$, based on the likelihood ratio test and likelihood function

$$
L\left(\beta_{1}, \beta_{2}\right)=\prod_{j=1}^{N_{1}+N_{f}} \frac{1}{1+\exp \left(\mu^{*}+\beta_{g i}^{*}\right)} \prod_{k=1}^{N_{f}} \exp \left(\mu^{*}+\beta_{g k}^{*}\right),
$$

is not rejected. Here $g j$ are genotypes of the diseased children and unrelated cases and $\beta_{i}^{*}, \mu^{*}$ are parameters defined in the Appendix. Let the p value of this likelihood ratio test be denoted as $P_{\mathrm{Com}}^{c a}$. We also conclude that the triads, unrelated cases and unrelated controls can be combined, if $\mathrm{p}$ values $P_{\mathrm{Com}}^{c o}$ and $P_{\mathrm{Com}}^{c a}$ are simultaneously greater than certain level, which will be defined later.

\section{The Second Stage Tests: Testing Association Based on Using}

Different Combinations of Genotype Data

In order to combine information from triads, unrelated cases and/or controls in a single analysis, Epstein et al. [10] considered an association analysis based on the likelihood of Nagelkerke et al. [9]. They suggested that powerful tests can be constructed based on the likelihoods depending on the products of the following four factors:

$$
L_{f}\left(\varphi_{1}, \varphi_{2}\right), \quad \prod_{i=1}^{N_{f}} P\left(G_{p i} \mid D_{i}=1 ; \varphi_{1}, \varphi_{2}, \mu\right), \quad \prod_{j=1}^{N_{0}} P\left(G_{j} \mid D_{j}=0 ; \mu\right),
$$

and

$$
\prod_{k=1}^{N_{1}} P\left(G_{k}=g_{k} \mid D_{k}=1 ; \varphi_{1}, \varphi_{2}, \mu\right) .
$$

The definitions of these probabilities can be found in Epstein et al. [10].

If the genotype data of triads and unrated controls are combinable, then they suggested the association test be defined as the likelihood ratio test, $T_{A s s o c}^{c o}$, for testing $H_{0}: \varphi_{1}=\varphi_{2}=0$ using likelihood function

$L^{c o}=L_{f}\left(\varphi_{1}, \varphi_{2}\right) \times \prod_{i=1}^{N_{f}} P\left(G_{p i} \mid D_{i}=1 ; \varphi_{1}, \varphi_{2}, \mu\right) \times \prod_{j=1}^{N_{0}} P\left(G_{j} \mid D_{j}=0 ; \mu\right)$.

We denote the $\mathrm{p}$ value of this test as $P_{A s s o c}^{c o}$. If the genotype data of triads and unrelated cases are combinable, then the association test is defined as the likelihood ratio test, $T_{A s s o c}^{c a}$, of $H_{0}$ using likelihood function

$$
\begin{aligned}
L^{c a}= & L_{f}\left(\varphi_{1}, \varphi_{2}\right) \times \prod_{i=1}^{N_{f}} P\left(G_{p i} \mid D_{i}=1 ; \varphi_{1}, \varphi_{2}, \mu\right) \\
& \times \prod_{k=1}^{N_{1}} P\left(G_{k}=g_{k} \mid D_{k}=1 ; \varphi_{1}, \varphi_{2}, \mu\right) .
\end{aligned}
$$

The $\mathrm{p}$ value of this test is denoted as $P_{A s s o c}^{c a}$. Finally, if the genotype data of triads and unrelated cases and controls are combinable, then the proposed association test is defined as the likelihood ratio test, $T_{A s s o c}^{c a+c o}$ of $H_{0}$ using likelihood function

$$
\begin{aligned}
L^{c o+c a}= & L_{f}\left(\varphi_{1}, \varphi_{2}\right) \times \prod_{i=1}^{N_{f}} P\left(G_{p i} \mid D_{i}=1 ; \varphi_{1}, \varphi_{2}, \mu\right) \\
& \times \prod_{j=1}^{N_{0}} P\left(G_{j} \mid D_{j}=0 ; \mu\right) \\
& \times \prod_{k=1}^{N_{1}} P\left(G_{k}=g_{k} \mid D_{k}=1 ; \varphi_{1}, \varphi_{2}, \mu\right) .
\end{aligned}
$$

We denote the $\mathrm{p}$ value of this test as $P_{\text {Assoc }}^{c a+c o}$.

The Two-Stage Test and Data-Dependent Significance Levels

We next show how to use $\mathrm{p}$ values, $P_{\mathrm{Com}}^{c a}$ and $P_{\mathrm{Com}}^{c o}$, at the first stage to guide the choice of association test at the second stage. Suppose that we have significance level $\alpha_{1}$ for the stage one tests and level $\alpha_{2}$ for the stage two tests. Then the second stage association test should be (1) test $T_{A s s o c}^{c o}$, if $P_{C o m}^{c o}>\alpha_{1}$, and $P_{C o m}^{c a} \leq \alpha_{1}$, (2) test $T_{\text {Assoc }}^{c a}$, if $P_{\text {Com }}^{c o} \leq \alpha_{1}$, and $P_{\text {Com }}^{c a}>\alpha_{1}$, (3) test $T_{\text {Assoc }}^{c a+c o}$, if $P_{\text {Com }}^{c o}>$ $\alpha_{1}$, and $P_{\mathrm{Com}}^{c a}>\alpha_{1}$, or (4) test $T_{C P G}$, if $P_{\mathrm{Com}}^{c o} \leq \alpha_{1}$, and $P_{\mathrm{Com}}^{c a} \leq \alpha_{1}$. As a consequence, the $\mathrm{p}$ value of the TS test is given by

$P=P_{\text {Assoc }}^{c o} I\left(P_{\text {Com }}^{c o}>\alpha_{1}, P_{\text {Com }}^{c a} \leq \alpha_{1}\right)+P_{\text {Assoc }}^{c a} I\left(P_{\text {Com }}^{c o} \leq \alpha_{1}, P_{\text {Com }}^{c a}>\alpha_{1}\right)$ $+P_{\text {Assoc }}^{c o+c a} I\left(P_{\text {Com }}^{c o}>\alpha_{1}, P_{\text {Com }}^{c a}>\alpha_{1}\right)+P_{C P G} I\left(P_{\text {Com }}^{c o} \leq \alpha_{1}, P_{\text {Com }}^{c a} \leq \alpha_{1}\right)$,

where $I(\cdot)$ is the usual indicator function taking values 0 or 1 .

We suggest a bootstrap method for choosing the significance levels $\alpha_{i}$ so that the overall type I error rate of the TS test always can be approximately controlled at some expected level $\alpha$. Let $P_{j}$ denote the p value of the TS test based on the $j$-th bootstrap sample, $j=1 \ldots$ B. Each bootstrap sample consists of a random sample of size $N_{f}$ collected from family triads, a random sample of size $N_{1}$ from unrelated cases and a random sample of size $N_{0}$ from unrelated controls. If the expected type I error rate of the TS test is $\alpha$, then we propose choosing $\alpha_{1}$ and $\alpha_{2}$ so that

$$
\left|B^{-1}\left\{\sum_{j=1}^{B} I\left(P_{i} \leq \alpha_{2}\right)\right\}-\alpha\right|
$$

is minimized. This is a general approach. A simpler but also reliable variation is to set significance level $\alpha_{2}=\alpha$ at the second stage, 
and so the minimization is taken only over all possible values of $\alpha_{1}$ between 0 and 1 . According to our experience in simulations, this approach would simplify the computation and make the performance of the TS test more stable. A free software (TS test software), based on the latter approach (setting $\alpha_{2}=\alpha$ ), for computing the data-dependent significance level at stage one, and the final $\mathrm{p}$ value of the TS test, is available at Cheng's software Web site.

\section{Simulations}

We have conducted several simulations to compare the performance of CPG and TS tests. Their performance depends on the number of family triads $N_{f}$ and the numbers of unrelated cases and controls, $N_{1}$ and $N_{0}$, the frequency of the high-risk allele, the level of genetic effect and the bias level of population stratification. According to equations (A3) and (A4) in the Appendix, the genotype frequencies of the data from family triads, unrelated cases and unrelated controls, depend on the genotype risks, mating type frequencies and the proportions of subpopulations collected in each substudy. In the simulations, we assumed that the general population comprises of two subpopulations, and HardyWeinberg equilibrium holds within each subpopulation. The high-risk allele frequency of the candidate marker in subpopulation 1 was $p=0.10$ or 0.30 and that in subpopulation 2 was $p+t$. A large difference, $t$, between the allele frequencies in the two subpopulations means that a large bias due to population stratification occurs in the usual case-control study. In the simulations, $t=0.03,0.05,0.07$ and 0.10 were considered. We also assumed that the family data were sampled from subpopulation 1 with proportion $q_{F}$ and from subpopulation 2 with proportion $1-q_{F}$. The unrelated cases and unrelated controls were sampled from subpopulation 1 with proportions $q_{C A}$ and $q_{C O}$, respectively. Four combinations of proportions were considered in the simulations. Model $(1)$ assumed $\left(q_{F}, q_{C A}, q_{C O}\right)=(0.6,0.6,0.6)$, representing the scenario of no effect of population stratification under null genedisease association; that is, family triads, unrelated cases and unrelated controls can be combined into a single analysis as we described. Model (2) assumed $\left(q_{F}, q_{C A}, q_{C O}\right)=(0.6,0.4,0.6)$, representing the scenario that family triads and unrelated controls are combinable. Model (3) assumed $\left(q_{F}, q_{C A}, q_{C O}\right)=(0.6,0.5$, 0.4 ), representing the scenario that there exist effects of population stratification and CPG test is employed for testing gene-disease association. Model (4) assumed $\left(q_{F}, q_{C A}, q_{C O}\right)=(0.6,0.6,0.4)$, representing the scenario that family triads and unrelated cases can be combined into a single analysis.

Finally, let $f_{s}(g)$ denote the genotype risk of a subject from subpopulation $\mathrm{s}$ and carries $g$ copies of high-risk allele. Under null simulations, identical risks $f_{1}(0)=f_{1}(1)=f_{1}(2)=f=0.05$ and $f_{2}(0)=f_{2}(1)=f_{2}(2)=0.10$ were used for subpopulation 1 and subpopulation 2 , respectively. In power simulations using the dominant genetic model, we assumed $f_{1}(0)=f_{1}, f_{1}(1)=f_{1}(2)=f_{1}+0.15$, $f_{2}(0)=0.10, f_{2}(1)=f_{2}(2)=f_{2}$ and considered $\left(f_{1}, f_{2}\right)=(0.15,0.20)$, and $(0.05,0.40)$, corresponding to the cases where relative risks $\left(\varphi_{1}, \varphi_{2}\right)=(\varphi, \varphi)$ respectively equal to $(2,2)$, and $(4,4)$ for both subpopulations. Under the recessive genetic model, we assumed $f_{1}(0)=f_{1}=f_{1}(1), f_{1}(2)=f_{1}+0.15, f_{2}(0)=0.10=f_{2}(1), f_{2}(2)=f_{2}$ and considered $\left(f_{1}, f_{2}\right)=(0.15,0.20)$, and $(0.05,0.40)$, corresponding to the cases with relative risks $\left(\varphi_{1}, \varphi_{2}\right)=(1, \varphi)=(1,2)$, and $(1,4)$, respectively. Under the additive genetic model, we assumed $f_{1}(0)=$ $f_{1}, f_{1}(1)=f_{1}+0.15, f_{1}(2)=f_{1}+0.30, f_{2}(0)=0.10$ and considered $\left(f_{1}, f_{2}(1), f_{2}(2)\right)=(0.15,0.20,0.30),(0.10,0.25,0.40)$ and $(0.05,0.40$,
$0.70)$, corresponding to the cases with relative risks $\left(\varphi_{1}, \varphi_{2}\right)=$ $(\varphi, 2 \varphi-1)$ equal to $(2,3)$, and $(4,7)$, respectively.

Under the given simulation conditions, we generated genotype data for family triads, unrelated cases and unrelated controls. The numbers of unrelated cases and controls were both equal to $100\left(N_{1}=100, N_{0}=100\right)$ and the number, $N_{f}$, of family triads was 60 or 120 . The $p$ values of the tests at stages one and two were all computed from chi-square distribution with 2 degrees of freedom. We used overall significance level $\alpha=0.05$ in all simulations. The number of bootstrap replications for computing data-dependent significance level at stage one was B = 100 (we had chosen significance level $\alpha_{2}=\alpha=0.05$ at stage two in all simulations). Estimates of type I error rates or powers were based on 2000 replications. Thus for a particular null (or power) simulation, each estimate of type I error rate (or power) was the proportion of the replicates for which the overall $p$ value was $\leq 0.05$.

\section{Results}

\section{Empirical Type I Error Rates}

Results for the simulated type I error rates are presented in figure 1 under the presence and absence of the effect of population stratification. The bias levels caused by population stratification can be indirectly measured by the differences between the simulated type I error rates of the likelihood ratio test of the case-control substudy (denoted as CC test) and 0.05. Recall that there is no effect of population stratification under Model 1. In this case, the unreported simulated type I error rates of the CC test range only from 0.045 to 0.065 . On the other hand, under models 2-4, there exists effect of population stratification and the range of the simulated type I error rates of the CC test is $(0.049,0.098)$. If one changes sampling proportions $q_{C A}$ from 0.5 to 0.7 and $q_{C O}$ from 0.4 to 0.3 in Model 3, the largest simulated type I error rate would change from 0.098 to 0.184 .

Next, inspecting the curves of the type I error rates, we find that the performance of the CPG and TS tests are both stable. Most of their type I error rates are very close to the expected 0.05 level under all simulation conditions. We note that the performance of the CPG test only depends on the allele frequency and sample size and is independent of simulation models. The overall range of the type I error rates of the CPG test is only $(0.040,0.065)$. However, we find out that the variation of its type I error rates are larger when the number of family triads becomes smaller. In contrast, the type I errors of the TS test range from 0.042 to 0.057 when allele frequency is 0.10 and from 0.040 to 0.051 when the allele frequency is 0.30 . In general, the performance of the TS test is more stable. 

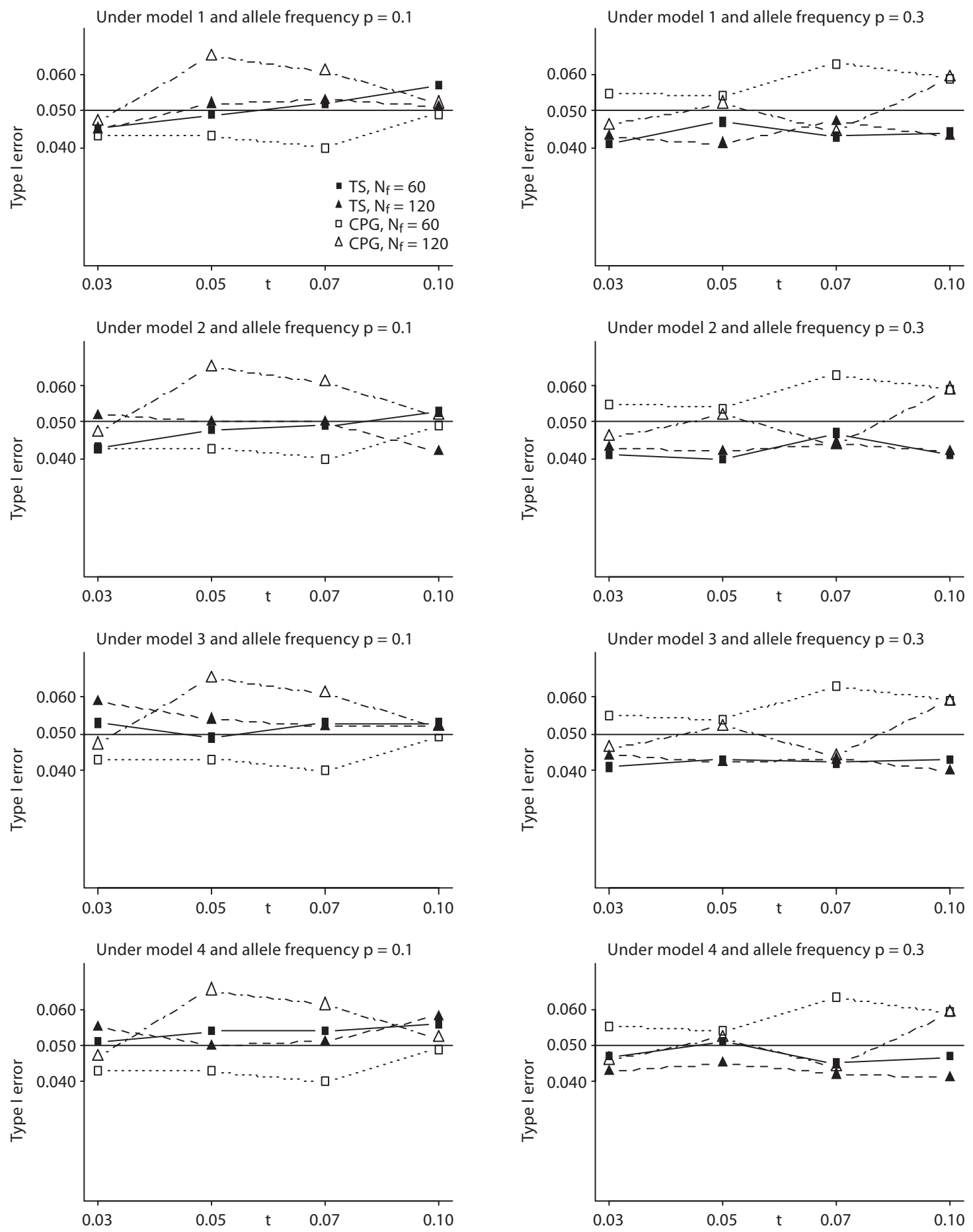

Fig. 1. Type I errors of the two-stage TS test and CPG test under the presence and absence of the effect of population stratification. 

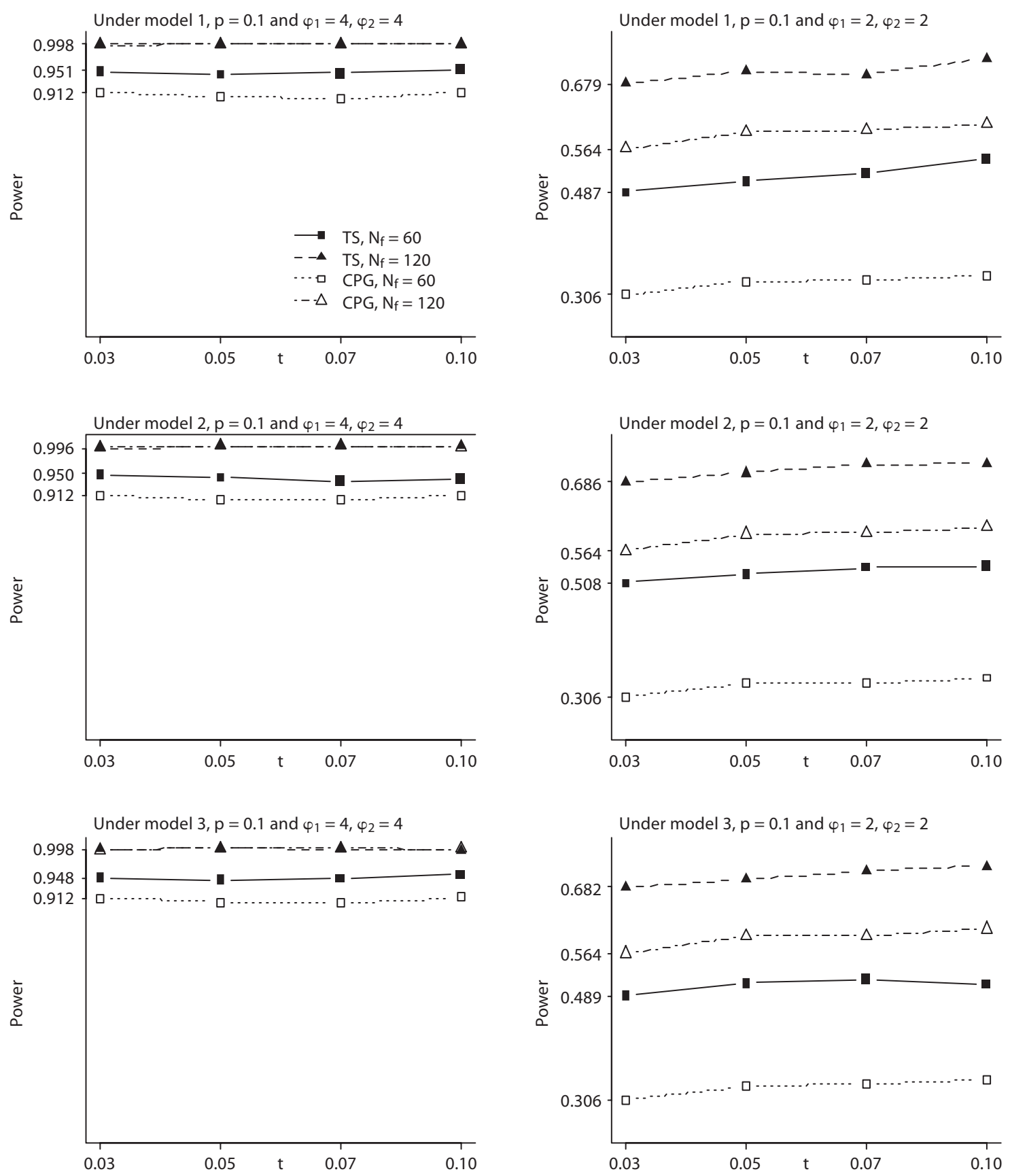


\section{Empirical Powers}

The simulated results about the powers of the CPG and TS tests under dominant genetic model are summarized in figures 2 and 3 . The simulated powers under the recessive and additive models can be found in supplemental material (see www.karger.com/doi/000228925). Inspecting the power results, we first find out the performance of the CPG and TS tests are both satisfactorily when the genetic effect is large and the genetic model is dominant or additive. For example, when the relative risk is $\varphi=4$, the range of the powers of the CPG test is $(0.887$, $0.999)$ and $(0.915,1.000)$ under the dominant genetic model and the additive genetic model, respectively. The corresponding ranges of the TS test are $(0.935,0.999)$ and $(0.941,1.000)$. Under the recessive genetic model (fig. 3 ), but larger allele frequency $(\mathrm{p}=0.30)$, and relative risk $(\varphi=4)$, the smallest power of the CPG test is 0.841 . In contrast, the smallest power of the TS test is 0.901 . However, under the same genetic model, high relative risk but small allele frequency $(\mathrm{p}=0.10)$, the performance of the CPG test deteriorates sharply. In this case, the largest power is only 0.355 when $N_{f}=60$ and 0.625 when $N_{f}=120$. In comparison, the largest powers of the TS test are 0.535 and 0.737 , respectively. In the same case, the largest (smallest) percentage increase of the power of the TS test over the CPG test is $57.95 \%$ (41.11\%) when $N_{f}=60$, and $25.34 \%(15.52 \%)$ when $N_{f}=120$.

The performance of the TS test is also substantially better than the CPG test when the relative genetic risk is low $(\varphi=2)$ and the genetic model is dominant or additive. When $N_{f}=60$, the smallest (largest) percentage of power increase of the TS test over the CPG test is 50\% (66\%) under the dominant genetic model and $34.09 \%$ (56.64\%) under the additive genetic model. When $N_{f}=120$, the smallest (largest) percentage of the power increase becomes $14.98 \%(21.63 \%)$ under the dominant genetic model and $6.38 \%(17.24 \%)$ under the additive model. In the same low relative risk case but under the recessive genetic model and high allele frequency $(\mathrm{p}=0.30)$, the smallest (largest) percentage of the power increase of the TS test is 58.03 (75.49\%) when $N_{f}=60$ and $23.67 \%$ (31.99\%) when $N_{f}=$ 120. If the frequency of the high-risk allele reduces to $\mathrm{p}=$ 0.10 , the power improvement of the TS test becomes marginal when the difference of allele frequencies $t$ is small. However, when the difference becomes large, the smallest (largest) percentage of power increase becomes $14.01 \%$ (39.8\%).

Comparing the performance of the TS test under three different models, we also find out that its power improvement over the CPG test does not differ significantly be- tween Models 1 and 2 and is only slightly smaller under Model 3. Also, the power improvement of the TS test is larger under the case of weak effect of population stratification. For example, under the dominant genetic model the largest percentage power increase of the TS test is $66.80 \%$ in the case of Model 1 (representing no effect of population stratification) and $75.49 \%$ in the case of Model 2 (representing weak effect of population stratification).

\section{Discussion}

Epstein et al. [10] emphasized that the difficult step in gene discovery is the gathering of genetic data and the best possible analysis should be used even if specialized software is required. We take the same viewpoint and have proposed an approach depending on the use of combinability tests in the first stage and association tests in the second stage. The proposed combinability tests are different from the combinability tests of Epstein et al. The latter tests are only designed for testing same relative risk parameters (not for testing equal genotype frequencies and equal relative genotype risks) of different data populations and thus they are indirect tests; see the related argument in Epstein et al. In contrast, the new combinability tests are direct tests. For example, in testing the combinability of genotype data from family triads and unrelated cases, the effect of heterogeneous populations is measured by

$$
\begin{aligned}
\beta_{g}^{*}=\left\{\delta_{g}(1)-\delta_{g}(2)\right\} & +\log \frac{P(G=g \mid D=0, S=1)}{P(G=g \mid D=0, S=2)} \\
& -\log \frac{P(G=0 \mid D=0, S=1)}{P(G=0 \mid D=0, S=2)},
\end{aligned}
$$

where

$$
\begin{aligned}
\delta_{g}(s)= & \log \left\{\frac{P(D=1 \mid G=g, S=s)}{P(D=0 \mid G=g, S=s)}\right. \\
& \left.\times \frac{P(D=0 \mid G=0, S=s)}{P(D=1 \mid G=0, S=s)}\right\}-\log \varphi_{g} .
\end{aligned}
$$

Thus any test for testing $\beta_{g}^{*}=0$ is a valid test of equal genotype frequencies and relative risks in populations 1 and 2; see (A2) of the Appendix. Unreported simulation results also indicate that the indirect test is often conservative and less efficient.

The two-stage test can be easily modified, if one has only genotype data from family triads and unrelated controls (cases). In this situation, one simply redefine $P_{\mathrm{Com}}^{c a}=$ $0\left(P_{\mathrm{Com}}^{c o}=0\right)$ in the definition of $\mathrm{p}$ value of the two-stage 


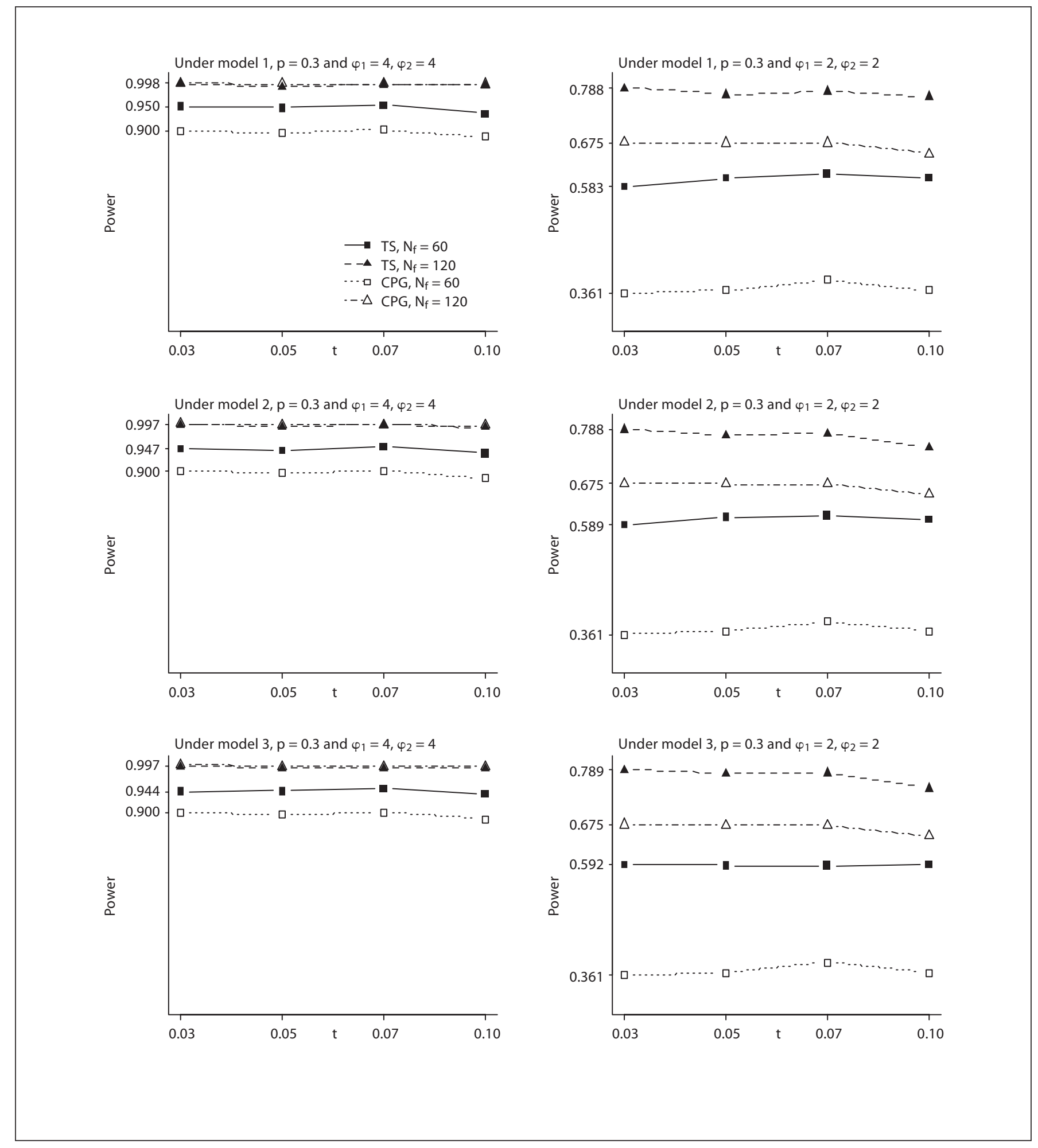

Fig. 3. Empirical powers of the TS and CPG tests under $\mathrm{p}=0.3$, dominant genetic model and different levels of population stratification. 
test. It also can be modified to account for missing parental genotypes. If missingness does not depend on the parental genotype, we may follow the approach of Weinberg [12] to recover the missing information in the first stage tests. Similarly, the modified association tests in the second stage of our test procedure can be done following the idea of Epstein et al. [10].

The two-stage procedure involves many correlated tests in the testing process and generally has difficulty of determining overall $\mathrm{p}$ value. In this paper, we have proposed a bootstrap method to control the overall probability of making any false positives in the study. We also have conducted a simulation study to provide evidence for supporting this approach. The simulation results show that the overall type I errors of the TS test are very close to the desired levels. It also makes fewer false negatives than the usual family-based association tests. Figure 1 only shows partial results of the simulation study. If significance level $\alpha$ is either 0.10 or 0.01 , similar results also can be observed. In addition, if the proportions $q_{C A}$ and $q_{C O}$ in Model 3 of the simulations were changed from 0.5 to 0.7 and 0.4 to 0.3 , respectively, then the level of population stratification became moderate (the largest type I error of the usual case-control test was 0.184), the type I errors of the TS test were still controlled at the expected level. Therefore, we can safely claim that the TS test has the ability of controlling type I error to the predetermined level under realistic situations. Wacholder et al. [13] argued that bias due to genetic population stratification is small or negligible; see also Evangelou et al. [14]. Therefore, our simulation results seem to be sufficient to justify the use of the TS test in real applications.

It may not be all that surprising that sometimes, the two-stage approach results in reduced power because one can see that identical information is treated differently in two stages. However, this is not the case for the TS test. Under the conditions considered in the simulation study, we have observed significant improvement of the TS approach over the CPG approach. Particularly, the power improvement of the TS test is much more significant when the CPG test has weak to moderate power. However, if the number of family trios increases, the improvement becomes smaller. This means that the two-stage approach is especially useful when one does not have sufficient number of family data.

We finally remark that in our simulation study we assumed that the population stratification was from the combination of data from discrete subpopulations. However, the arguments in the Appendix show that our combinability tests still work under admixtured populations.

\section{Acknowledgements}

This work was supported in part by the National Science Council of Taiwan. The authors also like to thank reviewers for their constructive comments which improve the presentation of the paper.

\section{Appendix}

Derivation of the Combinability Tests

Under structured population, we assume for simplicity of presentation that the family triads were sampled from population 1 , and unrelated cases and unrelated controls were sampled populations 2 and 3, respectively. (Each population can be described by a combination of $\mathrm{K}$ subpopulations as we shall see later.) Covariable $\mathrm{S}$ denotes the population to which a person belongs. Thus the relation between the genotype frequencies of the affected subjects (cases) in case-parents substudy and unrelated controls can be described by

$\operatorname{Pr}(G=g \mid D=1, S=1)=\operatorname{Pr}(G=g \mid D=0, S=3) \times \exp \left(\mu+\beta_{g}\right)$.

Here, $\mu$ is a normalizing constant and each log-odd-ratio parameter can be expressed as a sum of base-line log-relative-risk and bias $B_{g}$; that is $\beta_{g}=\log \varphi_{g}+B_{g}$. Bias $B_{g}$ is given by

$$
\begin{aligned}
& B_{g}=\log \left\{\frac{P(G=g \mid D=0, S=1) \exp \left(\alpha_{1}^{*}+\delta_{g}(1)\right)}{P(G=g \mid D=0, S=3)}\right\}- \\
& \log \left\{\frac{P(G=0 \mid D=0, S=1) \exp \left(\alpha_{1}^{*}+\delta_{0}(1)\right)}{P(G=0 \mid D=0, S=3)}\right\}, \\
& \alpha_{1}^{*}=\log \left\{\frac{P(D=1 \mid G=0, S=1)}{P(D=0 \mid G=0, S=1)}\right\}+\log \left\{\frac{P(D=0, S=1)}{P(D=1, S=1)}\right\}
\end{aligned}
$$

and

$$
\begin{aligned}
\delta_{g}(s)= & \log \left\{\frac{P(D=1 \mid G=g, S=s)}{P(D=0 \mid G=g, S=s)}\right. \\
& \left.\times \frac{P(D=0 \mid G=0, S=s)}{P(D=1 \mid G=0, S=s)}\right\}-\log \varphi_{g},
\end{aligned}
$$

which is the difference of the log-odds-ratio of population $s$ and log-relative-risk of population 1. Using equation (A1), it is well known that the likelihood of the data from all cases (in familybased substudy) and controls is proportional to

$$
L\left(\beta_{1}, \beta_{2}\right)=\prod_{j=1}^{N_{0}+N_{f}} \frac{1}{1+\exp \left(\mu+\beta_{g j}\right)} \prod_{k=1}^{N_{f}} \exp \left(\mu+\beta_{g k}\right),
$$

where $g j(g k)$ are genotypes of the subjects in the case and control sample (case sample). Since one shows that $B_{g} \approx 0$, if the disease of interest is rare and genotype frequencies of populations 1 and 3 are the same. Thus letting $\hat{\varphi}_{1}$, an $\mathrm{d} \hat{\varphi}_{2}$ be the maximum likelihood estimates using the 'conditional on parental genotypes' likelihood function $L_{f}\left(\varphi_{1}, \varphi_{2}\right)$, the likelihood ratio test for testing $H_{0}^{c o}: \beta_{1}=\log \hat{\varphi}_{1}, \beta_{2}=\log \hat{\varphi}_{2}$, is a valid test for testing the combinability of the case-parents and unrelated control data. 
Following the same principle, we define test for testing the combinability of family triads and unrelated cases. We treat the affected subjects in family triads as cases but treat unrelated cases as controls in the analysis. The relation between the genotype frequencies of the affected subjects (cases) in family triads and unrelated cases can be written as

$$
\begin{aligned}
\operatorname{Pr}(G=g \mid D=1, S=1)= & \operatorname{Pr}(G=g \mid D=1, S=2) \\
& \times \exp \left(\mu^{*}+\beta_{g}^{*}\right),
\end{aligned}
$$

where

$$
\begin{aligned}
& \beta_{g}^{*}=\left\{\delta_{g}(1)-\delta_{g}(2)\right\}+\log \frac{P(G=g \mid D=0, S=1)}{P(G=g \mid D=0, S=2)} \\
&-\log \frac{P(G=0 \mid D=0, S=1)}{P(G=0 \mid D=0, S=2)},
\end{aligned}
$$

and $\mu^{*}$ is a normalizing constant. Thus the likelihood of the data from all cases in family triads and unrelated cases is proportional to

$$
L^{*}\left(\beta_{1}^{*}, \beta_{2}^{*}\right)=\prod_{j=1}^{N_{1}+N_{f}} \frac{1}{1+\exp \left(\mu^{*}+\beta_{g j}^{*}\right)} \prod_{k=1}^{N_{f}} \exp \left(\mu^{*}+\beta_{g k}^{*}\right) .
$$

Under rare disease and if populations 1 and 2 have identical genotype frequencies and relative risks, then the above result implies that $\beta_{g}^{*} \approx 0$. This means that the likelihood ratio test for testing $H_{0}^{c a}: \beta_{1}^{*}=\beta_{2}^{*}=0$ is a valid test of the combinability of family triads and unrelated cases.

\section{Formulas for Generating Data from Family Triads and Unrelated Cases and Controls}

We assume that each population described above can be expressed as a combination of $\mathrm{K}(\mathrm{K}=2$ in the simulation study) subpopulations. We define $f_{s}(g)$ as the genotype risk of a subject who is from subpopulation $s$ and carries $g$ copies of high-risk allele. Let $P^{*}(S=s \mid D=d)$ denote the sampling proportion of subjects from subpopulation s with disease status $d, d=0$ or 1 . Since Mendelian proportions are assumed to be held within each subpopulation, one has genotype frequency from the controls given by

$$
\begin{aligned}
& P(G=g \mid D=0)= \\
& \sum_{s=1}^{K} \sum_{g_{p}} P\left(G=g, G_{p}=g_{p} \mid D=0, S=s\right) P^{*}(S=s \mid D=0)= \\
& \sum_{s=1}^{K} \sum_{g_{p}}\left(\frac{\left(1-f_{s}(g)\right) P\left(G=g \mid G_{p}=g_{p}\right) P\left(G_{p}=g_{p} \mid S=s\right) P^{*}(S=s \mid D=0)}{\sum_{g_{p}, g}\left(1-f_{s}(g)\right) P\left(G=g \mid G_{p}=g_{p}\right) P\left(G_{p}=g_{p} \mid S=s\right)}\right),
\end{aligned}
$$

where for each $\mathrm{s}$, the summation in the denominator is taken over all possible combinations of $\left(g, g_{p}\right)$. The genotype data of the cases and their parents can be generated from the joint frequencies

$$
\begin{aligned}
& P\left(G=g, G_{p}=g_{p} \mid D=1\right) \\
& =\sum_{s=1}^{K}\left(\frac{f_{s}(g) P\left(G=g \mid G_{p}=g_{p}\right) P\left(G_{p}=g_{p} \mid S=s\right) P^{*}(S=s \mid D=1)}{P(D=1 \mid S=s)}\right) \\
& =\varphi_{g} \sum_{s=1}^{K}\left(\frac{P\left(G=g \mid G_{p}=g_{p}\right) P\left(G_{p}=g_{p} \mid S=s\right) P^{*}(S=s \mid D=1)}{\sum_{g_{p}, g} \varphi_{g} P\left(G=g \mid G_{p}=g_{p}\right) P\left(G_{p}=g_{p} \mid S=s\right)}\right),
\end{aligned}
$$

where the last equality holds only when the genotype relative risks are homogeneous across subpopulations.

\section{Web Resource}

Software for computing the data-dependent significance level and $p$ value of the TS test is available at the following URL: http://www2.cmu.edu.tw/ biostat/downloads/KFCpro/KFC program.htm.

\section{References}

1 Falk CT, Rubinstein P: Haplotype relative risks: An easy reliable way to construct a proper control sample for risk calculations. Ann Hum Genet 1987;51:227-233.

2 Spielman RS, McGinnis RE, Ewens WJ: Transmission test for linkage disequilibrium: the insulin gene region and insulin-dependent diabetes mellitus (IDDM). Am J Hum Genet 1993;52: 506-516.

3 Ott J: Statistical properties of the haplotype relative risk. Genet Epidemiol 1989;6:127-130.

4 Terwilliger JD, Ott J: A haplotype-based 'halotype relative risk' approach to detecting allelic associations. Hum Hered 1992;42:337-346.

5 Ewens WJ, Spielman RS: The transmission /disequilibrium test: history, subdivision and admixture. Am J Hum Genet 1995;57:455-464.
6 Thomson G: Mapping disease genes: Familybased association studies. Am J Hum Genet 1995;57:487-498

7 Schaid DJ, Sommer SS: Genotype relative risks: methods for design and analysis of candidategene association studies. Am J Hum Genet 1993;53:1114-1126.

8 Mitchell LE: Relationship between case-control studies and transmission/disequilibrium test. Genet Epidemiol 2000;19:193-201.

9 Nagelkerke N, Hoebee B, Teunis P, Kimman T: Combining the transmission disequilibrium test and case-control methodology using generalized logistic regression. Eur J Hum Genet 2004;12:964-970.

10 Epstein M, Veal C, Trembath R, Barker J, Li C, Satten G: Genetic association analysis using data from triads and unrelated subjects. Am Hum Genet 2005;76:592-608.
-11 Ackerman H, Usen S, Jallow M, Sisay-Joof F, Pinder M, Kwiatowski DP: A comparison of case-control and family-based association methods: The example of sickle-cell and malaria. Ann Hum Genet 2005;69:559-565.

12 Weinberg CR: Allowing for missing parents in genetic studies of case-parents triads. Am J Hum Genet 1999;64:1186-1193.

13 Wacholder S, Rothman N, Caporaso N: Population stratification in epidemiologic studies of common genetic variants and cancer: quantification of bias. J Natl Cancer Inst 2000;92:11511158.

14 Evangelou E, Trikalinos TA, Salanti G, Ioannidis JPA: Family-based versus unrelated casecontrol designs for genetic association. PLoS Genetics 2006;2:1147-1155. 